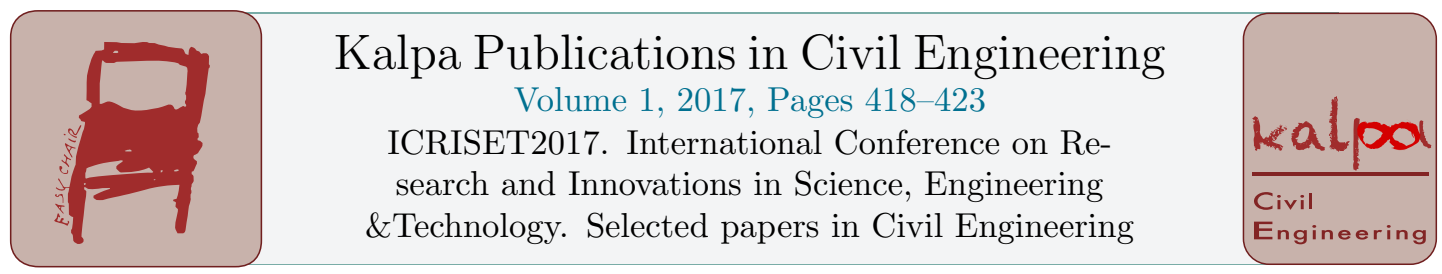

\title{
Development of Shock Spectra for Different Pulse Shapes Using C Sharp
}

\author{
Manav Patel ${ }^{1,2^{*}}$ and Dr. V. R. Panchal ${ }^{1 \dagger}$ \\ ${ }^{1}$ Civil and structural engineering, CHARUSAT, Changa, India \\ ${ }^{2}$ Professor and Head, CHARUSAT, Changa India \\ manav123.mp@gmail.com, vijaypanchal.cv@charusat.ac.in
}

\begin{abstract}
In this paper, study is carried out to develop the shock spectra for different impulse excitation. To evaluate the effect of damping on various shock spectra, SDOF Systems have been considered. Piece-wise differential equation method is used to evaluate the response of the systems. $0 \%, 5 \%, 10 \%$ and $20 \%$ damping ratios are considered for four different pulse excitation namely - First half triangular pulse, Second half triangular pulse, Full cycle sine wave pulse, Trapezoidal pulse. Software tool is developed to generate different shock spectra using C Sharp.
\end{abstract}

\section{Introduction}

Different kinds of loads like DL, WL, LL etc. are acting on building during its entire life period. The effect of these basic loads can be calculated and analyzed manually as well as using software. Due to increasing number of terrorist attacks and the accident accurse in laboratories, it is very important to analyze and design structure to be safer against the blast load. It is not mandatory that the Rectangular, Triangular and half-sine pulse forces can only act on any structure. Practically, a structure can be subjected to any arbitrary pulse but for our betterment, it is assumed to have some specific shape. During recent years, there were notable studies on the impact of rectangular, half sine waves, symmetrical triangular pulses. But till date very less research have been established on the analytical study of arbitrary pulses. Therefore in this study an attempt of evaluating impact of any arbitrary pulse on single degree of freedom system (SDOF) structure is made using C Sharp.

There are different methods to evaluate shock spectra for different types of pulse shape. For this research purpose, piece-wise differential equation is used to evaluate the same.

Kumar et al. (2014) ${ }^{[1]}$, have taken single degree of freedom system for study the effect of damping on shock spectra for three impulse excitation such as Triangular, half-cycle sine and Symmetrical triangular force pulses. To find the response of system they used Duhamel's integral method. They have been plotted Ten different time period for different frequency ratio. Generally the dynamic magnification factor (DMF) in the shock spectra is maximum for rectangular pulse followed by half-sine wave and

C.D. Modhera, G.J. Joshi, D. Soni, I.N. Patel, A.K. Verma, L.B. Zala, S.D. Dhiman, D.R. Bhatt, J.M. Rathod, B.C. Goradiya, M.S. Holia and D.K. Patel (eds.), ICRISET2017 (Kalpa Publications in Civil Engineering, vol. 1), pp. 418-423 
Symmetrical triangular pulse. After the study they found similar trend for the system with using damper. The method which is used to develop shock spectra is given below.

\section{Piece-wise Exact differential method}

The least difficult well-ordered method for analysis of SDOF system is alleged as "Piece wise exact differential" method, which is based on the exact solution of the equation of motion for response of a linear structure to a loading that varies linearly during a discrete time interval. In this method, the loading history is divided into time intervals.

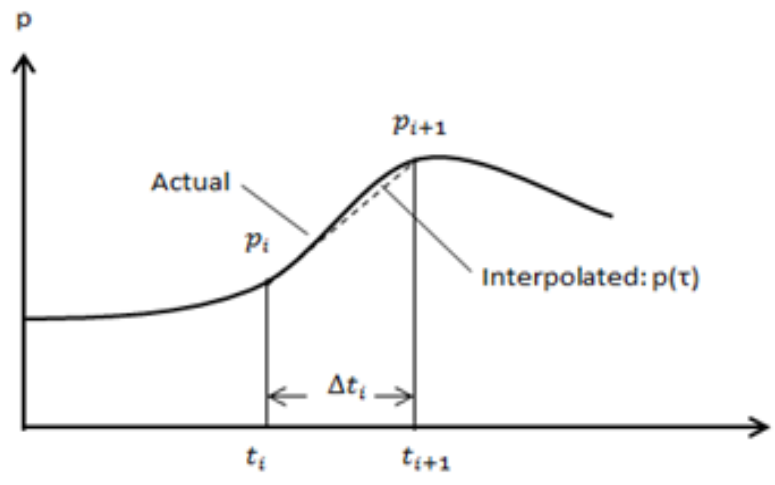

Figure 1: Notation for linearly interpolated excitation

Recurrence formula $^{[3]}$ is derived from the exact solution of equation of motion. The Recurrence formula for Displacement and velocity at time $t_{i+1}$ are given below,

$$
\begin{aligned}
& x_{i+1}=A x_{i}+B \dot{x}_{\imath}+C p_{i}+D p_{i+1} \\
& x_{i+1}=A^{\prime} x_{i}+B^{\prime} \dot{x}_{l}+C^{\prime} p_{i}+D^{\prime} p_{i+1} \\
& A=e^{-\zeta \omega_{n} \Delta \mathrm{t}}\left(\frac{\zeta}{\sqrt{1-\zeta^{2}}} \sin \omega_{D} \Delta \mathrm{t}+\cos \omega_{D} \Delta \mathrm{t}\right) \\
& B=e^{-\zeta \omega_{n} \Delta \mathrm{t}}\left(\frac{1}{\omega_{D}} \sin \omega_{D} \Delta \mathrm{t}\right) \\
& C=\frac{1}{k}\left\{\frac{2 \zeta}{\omega_{n} \Delta \mathrm{t}}+e^{-\zeta \omega_{n} \Delta \mathrm{t}}\left[\left(\frac{1-2 \zeta^{2}}{\omega_{D} \Delta \mathrm{t}}-\frac{\zeta}{\sqrt{1-\zeta^{2}}}\right) \sin \omega_{D} \Delta \mathrm{t}-\left(1+\frac{2 \zeta}{\omega_{n} \Delta \mathrm{t}}\right) \cos \omega_{D} \Delta \mathrm{t}\right]\right\} \\
& D=\frac{1}{k}\left[1-\frac{2 \zeta}{\omega_{n} \Delta \mathrm{t}}+e^{-\zeta \omega_{n} \Delta \mathrm{t}}\left(\frac{2 \zeta^{2}-1}{\omega_{D} \Delta \mathrm{t}} \sin \omega_{D} \Delta \mathrm{t}+\frac{2 \zeta}{\omega_{n} \Delta \mathrm{t}} \cos \omega_{D} \Delta \mathrm{t}\right)\right] \\
& A^{\prime}=-e^{-\zeta \omega_{n} \Delta \mathrm{t}}\left(\frac{\omega_{n}}{\sqrt{1-\zeta^{2}}} \sin \omega_{D} \Delta \mathrm{t}\right) \\
& B^{\prime}=-e^{-\zeta \omega_{n} \Delta \mathrm{t}}\left(\cos \omega_{D} \Delta \mathrm{t}-\frac{\zeta}{\sqrt{1-\zeta^{2}}} \sin \omega_{D} \Delta \mathrm{t}\right) \\
& C^{\prime}=\frac{1}{k}\left\{-\frac{1}{\Delta t}+e^{-\zeta \omega_{n} \Delta \mathrm{t}}\left[\left(\frac{\omega_{n}}{\sqrt{1-\zeta^{2}}}-\frac{\zeta}{\Delta t \sqrt{1-\zeta^{2}}}\right) \sin \omega_{D} \Delta \mathrm{t}-\frac{1}{\Delta t} \cos \omega_{D} \Delta \mathrm{t}\right]\right\} \\
& D^{\prime}=\frac{1}{k \Delta t}\left[1-e^{-\zeta \omega_{n} \Delta \mathrm{t}}\left(\frac{\zeta}{\sqrt{1-\zeta^{2}}} \sin \omega_{D} \Delta \mathrm{t}+\cos \omega_{D} \Delta \mathrm{t}\right)\right]
\end{aligned}
$$




\section{Procedure to develop Shock Spectra}

To develop response spectra, program has been made using C Sharp. This program is made in two phases. In first phase, using parameters such as Force $\left(\mathrm{P}_{0}\right)$, Pulse duration $\left(\mathrm{t}_{\mathrm{d}}\right)$, Time step $(\Delta \mathrm{T})$ pulse data are generated. In second phase of this program, Shock spectra is developed by using Piece-wise differential equation method. This method is explained in [2] part of introduction. From the piece-wise exact differential method maximum values of Dynamic displacement for different time ratio $\left(t_{d} / T_{n}\right)$ are obtained. Dividing dynamic displacement by static displacement deformation response factor $R_{d}$ $\left(x_{\max } / x_{\text {st } 0}\right)$ is obtained. Now ploting $\mathrm{R}_{\mathrm{d}}$ vs time ratio $\left(\mathrm{t}_{\mathrm{d}} / \mathrm{T}_{\mathrm{n}}\right)$ the graph obtained is known as Shock response spectra.

\section{Numerical study and Results}

Using above method four shock spectra has been developed. Shock spectra plotted for damped and undamped SDOF system for different pulse shapes taking damping ratios, $\xi=0 \%, 5 \%, 10 \%, 20 \%$. Following are some parameters considered for all four pulse shapes.

Force $\left(\mathrm{P}_{0}\right)=2.24 \mathrm{kN}$, Pulse duration $\left(\mathrm{t}_{\mathrm{d}}\right)=2.5 \mathrm{sec}$, Time step $(\Delta \mathrm{T})=0.01 \mathrm{sec}$, Mass $(\mathrm{m})=2243.85 \mathrm{~N}$ $\mathrm{sec}^{2} / \mathrm{m}$, Time step $\left(\mathrm{T}_{0}\right)=0.01 \mathrm{sec}$.

\subsection{First half - triangular pulse}

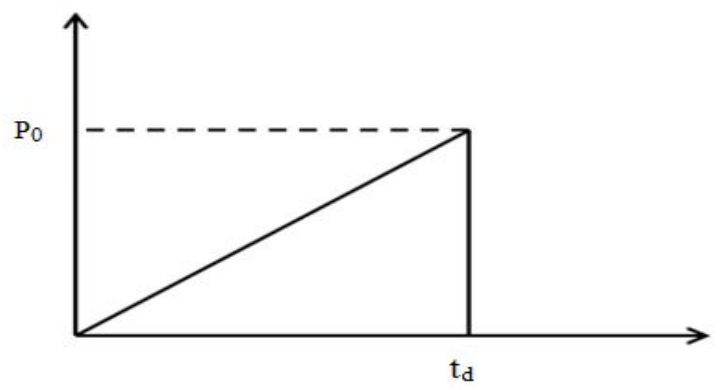

Figure 2: First half-triangular pulse

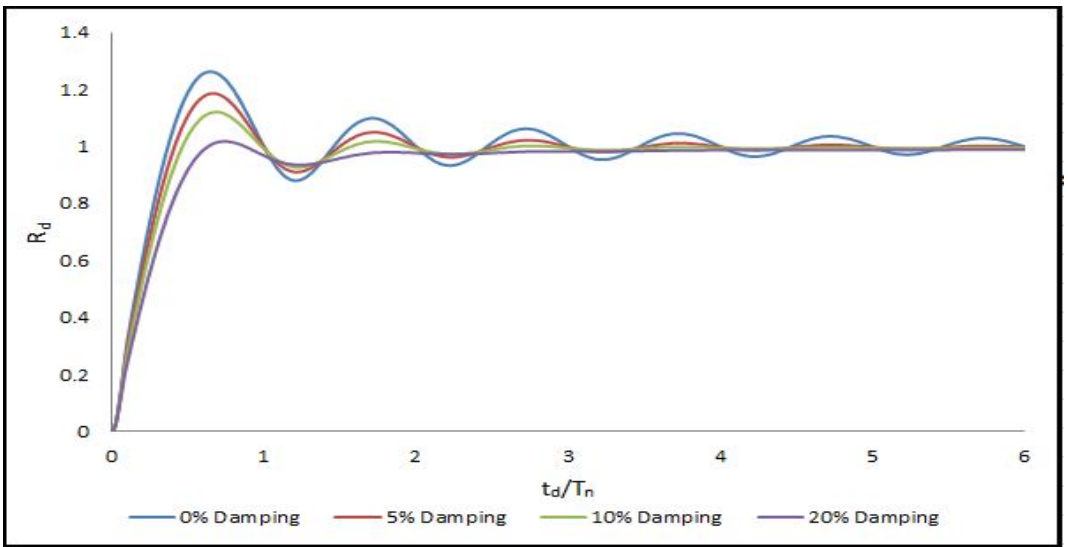

Figure 3: Shock spectra of first half - triangular pulse 


\subsection{Second half- triangular pulse}

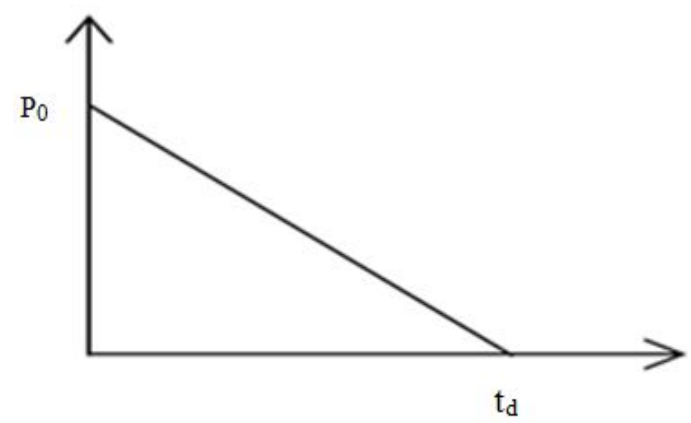

Figure 4: Second half-triangular pulse

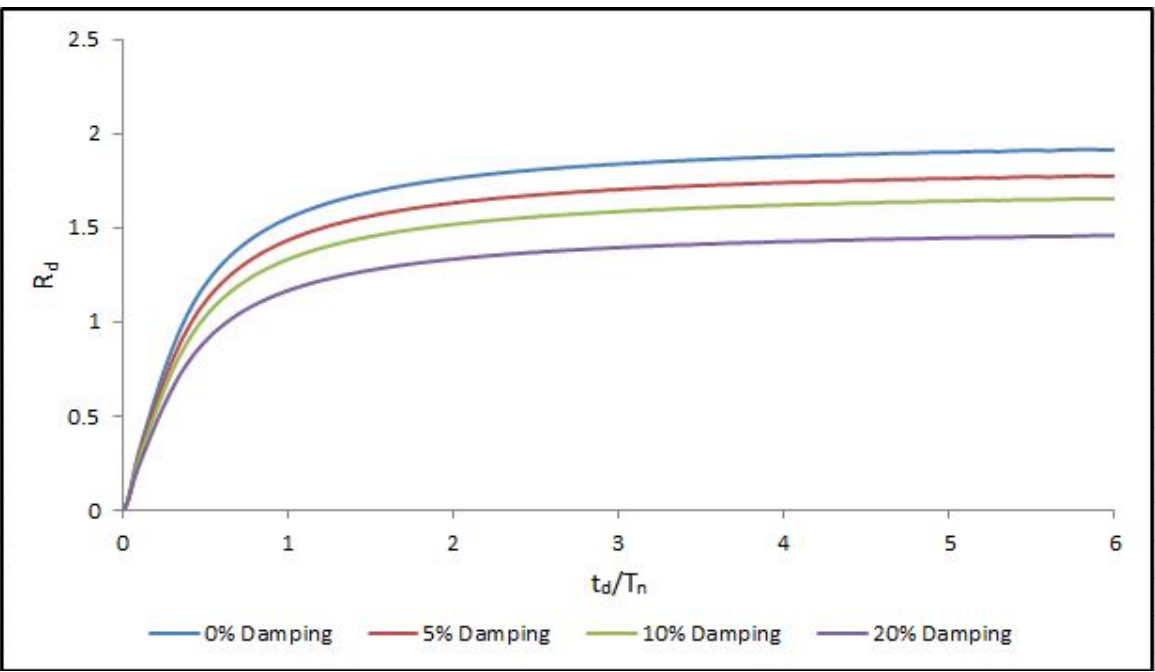

Figure 5: Shock spectra of second half-triangular pulse

\subsection{Full wave sine pulse}

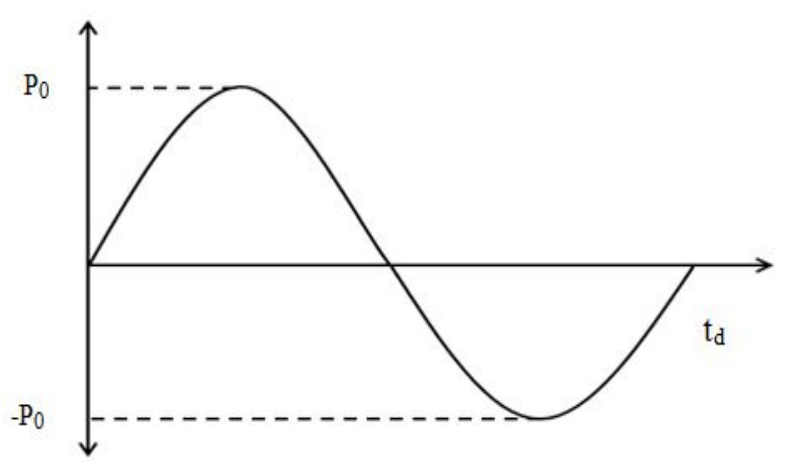

Figure 6: Full wave sine pulse 


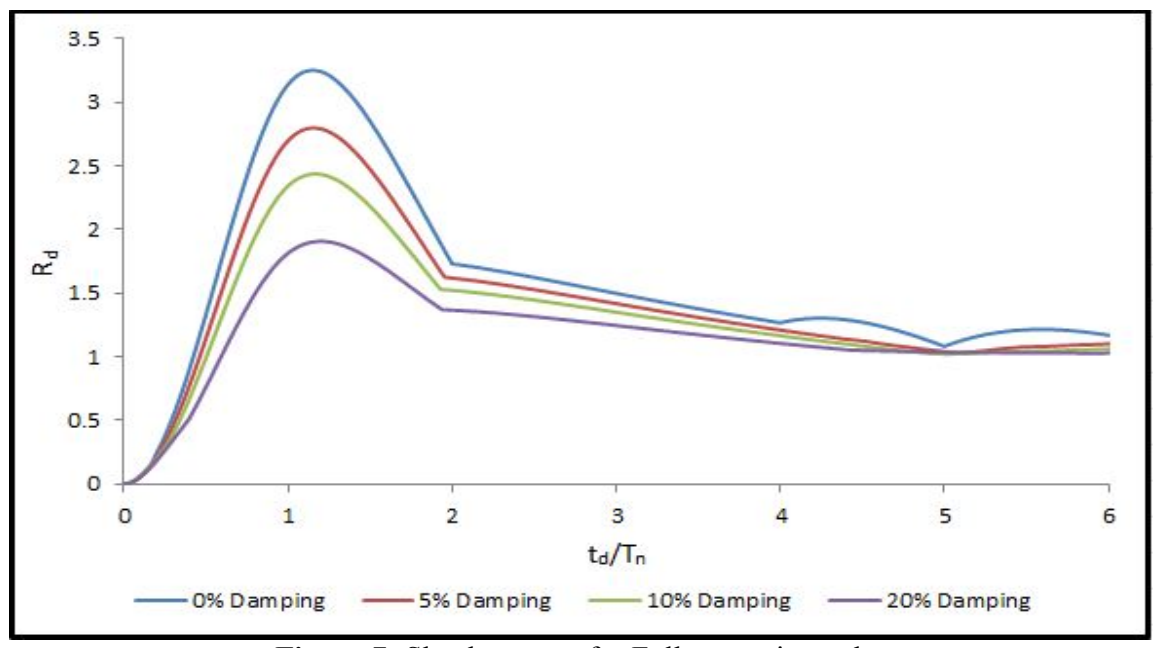

Figure 7: Shock spectra for Full wave sine pulse

\subsection{Trapezoidal pulse}

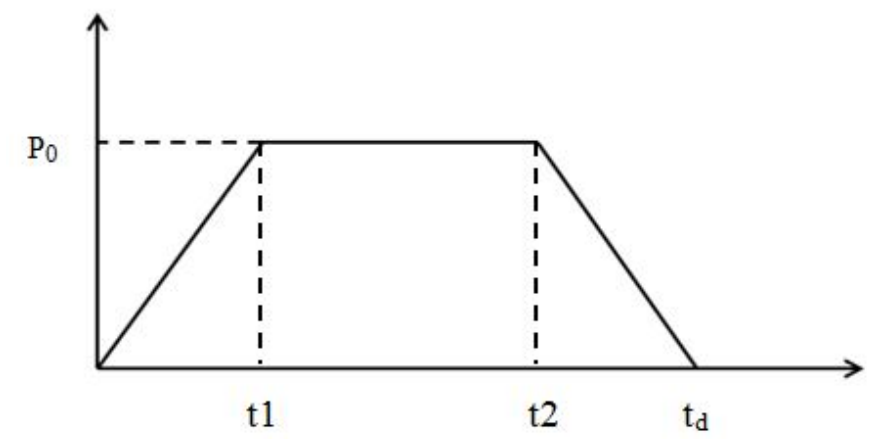

Figure 8: Trapezoidal pulse

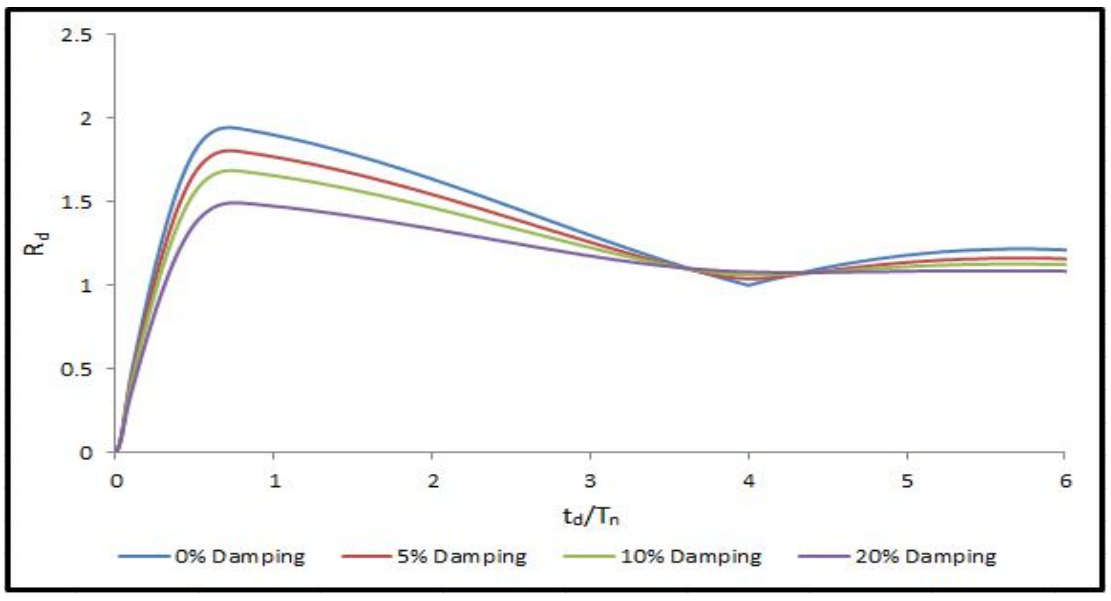

Figure 9: Shock spectra for Trapezoidal pulse 


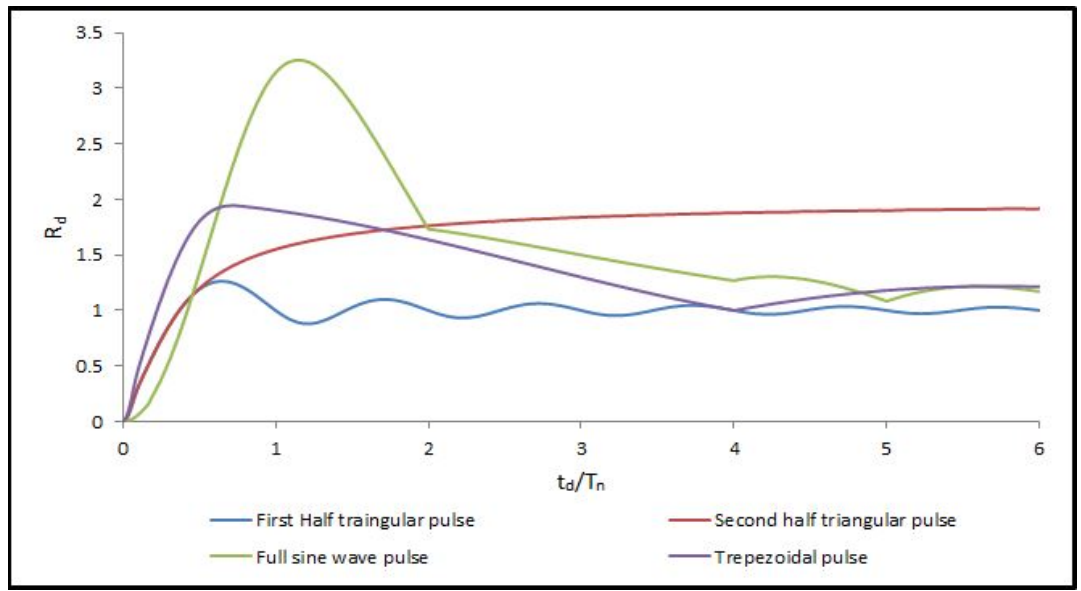

Figure 10: Shock spectra of different pulse of same amplitude $(\zeta=0 \%)$

\section{Conclusion}

1. Shock spectra for different pulses are developed.

2. For a given value of amplitude of impulse load, DMF is the maximum for Full sine wave pulse followed by Trapezoidal pulse, second half triangular pulse and first half triangular pulse.

3. Increase in damping reduces the DMF values for all impulse loads. Reduction seems proportion for change in damping.

\section{References}

Anil Kumar, P. A. (May-2014). Effect of Damping on Shock Spectra of Impulse Loads. International Journal of Scientific \& Engineering Research, Volume 5, Issue 5.

Chopra, A. K. ( 2011 ). Dynamics of Structures: Theory and Applications to Earthquake Engineering. Kelly, S. G. (2012). Mechanical Vibrations: Theory and Applications.

Paz, M. (2006). Structural Dynamics: Theory and Computation. CBS Publishers.

Penzien, R. C. (2012). Dynamics of structures. US: McGraw-Hill Inc.

Thomson, W. (1981). Theory of vibration with Applications. New Jersey. 